\title{
Assessment of quasi-linear effect of RF power spectrum for enabling lower hybrid current drive in reactor plasmas
}

\author{
Roberto Cesario ${ }^{1}$, Alessandro Cardinali ${ }^{1}$, Carmine Castaldo ${ }^{1}$, Luca Amicucci ${ }^{2}$, Silvio Ceccuzzi $^{1}$, Alessandro Galli ${ }^{2}$, \\ Francesco Napoli ${ }^{1}$, Luigi Panaccione ${ }^{1}$, Franco Santini ${ }^{1}$, Giuseppe Schettini ${ }^{3}$, Angelo Antonio Tuccillo ${ }^{1}$ \\ ${ }^{1}$ ENEA Fusion and Nuclear Safety Department, C. R. Frascati, Via E. Fermi 45, 00044 Frascati (Rome) Italy \\ ${ }^{2}$ Università Roma La Sapienza, Dipartimento di Ingegneria, Rome Italy \\ ${ }^{3}$ Università Roma Tre, Dipartimento di Ingegneria, Rome, Italy
}

\begin{abstract}
The main research on the energy from thermonuclear fusion uses deuterium plasmas magnetically trapped in toroidal devices. To suppress the turbulent eddies that impair thermal insulation and pressure tight of the plasma, current drive (CD) is necessary, but tools envisaged so far are unable accomplishing this task while efficiently and flexibly matching the natural current profiles self-generated at large radii of the plasma column [1-5]. The lower hybrid current drive (LHCD) [6] can satisfy this important need of a reactor [1], but the LHCD system has been unexpectedly mothballed on JET. The problematic extrapolation of the LHCD tool at reactor graded high values of, respectively, density and temperatures of plasma has been now solved. The high density problem is solved by the FTU (Frascati Tokamak Upgrade) method [7], and solution of the high temperature one is presented here. Model results based on quasi-linear (QL) theory evidence the capability, w.r.t linear theory, of suitable operating parameters of reducing the wave damping in hot reactor plasmas. Namely, using higher RF power densities [8], or a narrower antenna power spectrum in refractive index $[9,10]$, the obstacle for LHCD represented by too high temperature of reactor plasmas should be overcome. The former method cannot be used for routinely, safe antenna operations, Thus, only the latter key is really exploitable in a reactor. The proposed solutions are ultimately necessary for viability of an economic reactor.
\end{abstract}

\section{Introduction}

The main research on fusion energy utilises toroidal plasmas magnetically trapped in toroidal machines (tokamaks). As major scientific problem for an economic reactor, we must understand how to lead strongly heated plasma to sustain a high fusion gain, while a large fraction of current is self-produced via pressure gradient naturally present especially at large radii of the plasma column.

Strong powers of heating and current drive (CD) injected into the plasma ensure that the current induced by the transformer continues to flow, maintaining high fusion gain required for energy production [9]. The huge costs of the heating and $\mathrm{CD}$ systems should be reduced by exploiting, via particle transport (bootstrap) effect, a strong fraction of current that the plasma steadily selfproduces, in the presence of pressure gradient across the plasma column mostly occurring at large radii [1]. Consequently, the pressure profile should be mandatorily controlled (for at least several hours for enabling energy production) by properly shaping the plasma current density radial profile, $j(r)$. This allows indeed suppressing turbulence that impairs thermal insulation and pressure tight of the plasma column, which in turn enables sustaining the plasma current.
Current drive (CD) is necessary for turbulence suppression [2-4], but tools envisaged so far are unable accomplishing this task, while efficiently and flexibly matching the natural self-generated current profiles [1]. The lower hybrid current drive (LHCD) [5] can satisfy this need, but, although considered long time ago as a major breakthrough for a reactor, this tool has been mothballed on JET and not considered so far a priority for ITER. This should represent a serious mistake because LHCD offers the peculiar feature of matching the self-produced current profiles by the plasma [1]. LHCD is based on the use of strong radio-frequency (RF) power coupled to lower hybrid (LH) plasma waves. They are efficiently damped via Landau wave-particle resonance (in the phase and drifting velocities, respectively) with a tail of the electron distribution function (EDF) [11]:

$u_{\Phi / /} \equiv \frac{\omega_{0}}{k_{/ /}}=\frac{c}{n_{/ /}} \approx 3 u_{\text {the }}$

where: $u_{\text {w// }}$ and $k_{/ / /}$are, respectively, the components of wave phase velocity and wavevector parallel to $\mathbf{B}_{0}, c$ is light speed, $n_{/ /} \equiv \frac{c k_{/ /}}{\omega_{0}} \quad$ is the parallel refractive index 
component, and $u_{\text {the }}=\sqrt{\frac{2 k_{B} T_{e}}{m_{e}}}$ is the electron thermal velocity, where $k_{\mathrm{B}}$ is the Boltzmann constant, $T_{\mathrm{e}}$ is the electron temperature, and $m_{\mathrm{e}}$ is the electron mass.

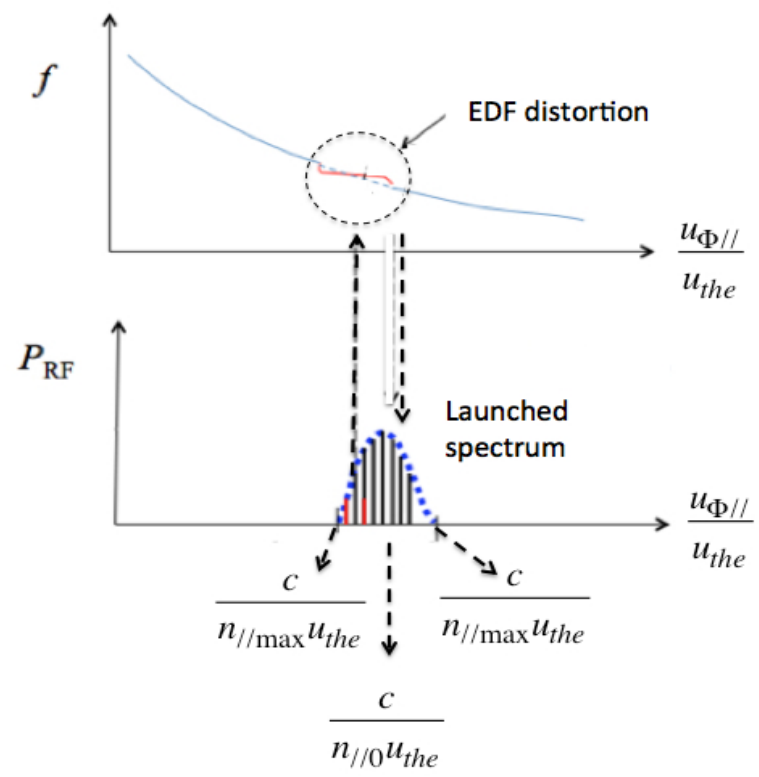

Fig. 1.

Top: electron distribution function (EDF). Bottom: Gaussian antenna spectrum of multi-megawatt radio-frequency power (in arbitrary units), coupled to plasma at several gigahertz, useful for producing the LHCD effect.

Accordingly to Eq. 1, plasma electrons Landau-resonate more intensely with spectral components with slower phase velocity. LH waves are electron plasma modes particularly prone to drive current, i.e, to transfer energy and momentum to plasma electrons along the direction of the confinement magnetic field, where they are free to drift. Consequent to slow-wave behaviour of LH modes (which have indeed refractive index component: $n_{/ /}>1$ ), electrons flowing with speed close to the phase velocity of the wave see its electric field as almost constant. The particles can thus surf on the wave, which results, via collisions, in an irreversible transfer of wave energy and momentum to the electrons. This produces the LHCD effect.

Consider, in the bottom box of the figure, the $n_{/ /}$power spectrum of LH waves launched by the antenna, centred at $n_{/ / 0}$, with spectral components in the interval $\left(n_{/ / \max }-n_{/ / \min }\right)$. Spreading radially towards the warmer regions of the plasma, the slowest components of the spectrum (with $u_{\mathrm{q} / / \sim u_{4} / / \min }$ i.e., $n_{/ /} \sim n_{/ / \max }$ ) are beginning to first to interact more intensely with the plasma, in accordance with Eq. 1. Consequent to accelerated population, some EDF flattening occurs as shown in the top box of the figure (and demonstrated by analytical model of Sec. 2).

This distortion determines the modality of the wave packet absorption and evidences the role of the spectral width ${ }^{7}$.

Figure 1 schematizes the LHCD concept [5], proven by experiment on PLT (Princeton Large Torus) [12], and successfully extrapolated on FTU (Frascati Tokamak Upgrade) to reactor-graded, high plasma densities [7,13]. The latter method was established following the guidelines of previous theoretical prediction of reduced parasitic wave interaction at the plasma edge under higher temperature of the latter $[14,15]$.

This can be accomplished by proper numerical tool able to display aspects of LHCD mechanism remained hidden so far, namely, in regard to the role of the width of the launched antenna power spectrum in the waveleght domain in determining the $j_{\mathrm{LH}}$ profile in reactor relevant condition of hot plasma that deterines full RF power absorption at single half of radial pass. Too hot plasma envisaged even at large radius of pedestal represents a major problem for LHCD exploitation in a reactor [16]. Here we present model results based on quasi-linear theory, evidencing the role, w.r.t linear theory, of reducing the wave damping via high RF power densities, as previously reported [6]. The latter option cannot be exploited for removing the obstacle represented by too high temperatures because too high RF power density should be required, which exceeds the power handling capability of antenna [17]. A useful way should therefore be considered, as recently proposed $[7,8]$. To solve the remaining major conceptual problem of LHCD, consisting in how to enable this effect in fusion relevant condition of high plasma temperature is ultimately necessary for viability of an economic reactor.

\section{Results}

\subsection{Analytical model}

We summarise here the wave damping analysis in 1-D quasilinear approximation. Consider the Gaussian spectrum with $n_{/ / 0}$ and $\Delta n_{/ /}$parameters ( $\Delta n_{/ /}$is kept at half of the peak value). Following QL theory [18], competing effects are produced: from one side, by the LH waveparticle interactions, Eq. 1, and related diffusion in the velocity space; from the other side, by collisions that tend restoring Maxwellian EDF. We analyse hereafter the condition for the EDF plateau formation, and show the link of wave packet with spectrum, schematized in Fig. 1. Assume slab plasma geometry where density and temperature gradients are directed along the $x$-direction defined above. In steady-state conditions, the spectral components $P\left(n_{\|}\right)$of the LH wave power are absorbed accordingly with the equations [19]:

$$
\frac{d P}{d x}=\Gamma_{Q L} P
$$

where $\Gamma_{\mathrm{QL}}$ is the quasilinear damping rate inferred from the 1-D distribution function of plasma electrons, and depending on the local RF power spectral density.

Following Landau [20] and considering the steady-state solution of the Fokker-Planck equation $[21,22]$ in the limit of LH waves, $\left|k_{\perp}\right|>>\left|k_{/ /}\right|$, the power damping can be approximated as [9]:

$$
\Gamma_{Q L} \approx \frac{\sqrt{\pi}}{k_{\perp} u_{\text {the }}^{2}} \omega_{p e}^{2} \frac{u_{0}}{1+u_{0}^{3} D_{Q L}\left(u_{0}\right)} e^{-u_{1}^{2}}
$$


where: $\quad u_{0} \equiv \frac{c}{n_{/ / 0} u_{\text {the }}}, \quad u_{1} \approx \frac{c}{\left(n_{/ / 0}+2 \Delta n_{/ /}\right) u_{\text {the }}}$

and

$D_{Q L}\left(u_{0}\right)$ is given by the expression [9]:

$$
D_{Q L}\left(u_{0}\right) \approx \frac{9}{\left(Z_{i}+2\right) \ln \Lambda^{e e}} \frac{\sqrt{T e[k e V]}}{\left(n_{e}\left[10^{20} m^{-3}\right]\right)^{\frac{3}{2}}} \frac{n_{/ / 0}^{2}-1}{\Delta n_{/ /}} p_{R F}\left[\frac{M W}{m^{2}}\right]
$$

In Eq. $4, Z_{\mathrm{i}}$ is the effective ion charge, $\ln \Lambda^{\mathrm{ee}}$ is the Coulombian logarithm that considers the electronelectron collision effect, and $p_{\mathrm{RF}}$ is the total power density of the spectrum.

Eq. 4 represents the QL diffusion coefficient normalized to the collision operator, both calculated at the centre of the EDF plateau (i.e., for $u_{/ /}=u_{0 / /}$ ). The level of the plateau is fixed by the exponential of Eq. 3 via the value: $n_{/ / \max } \approx\left(n_{0 / /}+2 \Delta n_{/ /}\right)$. This is the highest value of the launched spectrum (see Fig. 1) where $D(u)$ falls below one from values typically higher than 1000 in the plateau.

Neglecting QL diffusion, i.e. assuming an EDF of Maxwellian shape, the absorption coefficient in the linear wave limit is:

$$
\Gamma_{L} \approx \frac{\sqrt{\pi}}{k_{\perp} u_{\text {the }}^{2}} \omega_{p e}^{2} u_{0} e^{-u_{0}^{3}}
$$

Hence, the ratio of the QL and linear damping coefficients is:

$$
\frac{\Gamma_{Q L}}{\Gamma_{L}} \approx \frac{e^{\left(\frac{m_{e} c^{2}}{k_{B} T_{e}}\right)\left(\frac{2 \Delta n_{\| /}}{n_{/ /}^{3}}\right)}}{1+u_{0}^{3} D_{Q L}\left(u_{0}\right)}<1
$$

The inequality $\Gamma<\Gamma_{M}$ holds for:

$$
\Delta n_{/ /}<\frac{k_{B} T_{e}}{m_{e} c^{2}} n_{/ / 0}^{3} \ln \left(1+u_{0}^{3} D_{Q L}\left(u_{0}\right)\right)
$$

Condition of Eq.7 is widely verified for practical cases, i.e.: $\Delta n_{/ /} \leqslant 0.5, T_{\mathrm{e}} \sim 10 \mathrm{keV}, n_{\mathrm{e}} \sim 10^{20} \mathrm{~m}^{-3}, p_{\mathrm{RF}} \sim 30 \mathrm{MW} / \mathrm{m}^{2}$, $n_{/ / 0} \sim 1.8$, and $Z_{\mathrm{i}} \approx 1$.

For given plasma parameters, Eq. 6 shows conditions that QL physics effect is useful for reducing the RF power damping w.r.t. case of linear wave approximation. In the same framework of QL limit, Eq.4 and Eq.5 indicate that, for a certain $T_{\mathrm{e}}$, the wave damping should be reduced by means of higher values of $p_{\mathrm{RF}}$, as already reported [13]. This option is however prevented by the need of operating in safe operation compatible with the routinary power handling capability of antenna [14].

Importantly, Eq. 6 shows that a smaller $\Delta n_{/ /}$value pays a major role in reducing the RF power damping, owing to the exponential dependence in Eq. 5.

Numerical approach allows retaining effects of RF diffusion in 2-D dimensions in $u_{/ /}$and $u_{\perp}$ of electron velocity, useful for taking into account important details of the quasilinear diffusion coefficient, neglected in too rough approximation of Eq. 4, but necessary for assessing the LHCD profiles in practical cases.

\subsection{Summary of the numerical model}

The $\mathrm{LH}^{\text {star }}$ package of numerical codes $[13,7,8]$ has been revealed fully suitable for evidencing the QL physics shown in Sec. 2.1, namely, the effect of reduction of the RF power damping w.r.t. case of linear wave approximation, under higher values of higher $p_{\mathrm{RF}}$ or smaller $\Delta n_{/ /}$, for given reactor plasma parameters $[7,8]$. Differently from other numerical approaches $[19,23]$, the $\mathrm{LH}^{\text {star }}$ tool, in order to assess the $j_{\mathrm{LH}}$ profiles, treats the intrinsically LHCD single pass regime of reactor in simpler way because not affected by complicated architectures seeking to consider multi-pass regime, conceived with the aim of considering the case of LHCD experiments in experiments performed so far in less warm plasmas.

Consequenlty, by using antenna spectra sufficiently narrow and however compatible with constraints imposed by QL theory (trapping effect) it is possible enabling the LHCD effect and shaping the profile, spanning over the whole outer radial half of plasma of DEMO $[7,8]$.

More specifically, assuming linear wave theory, Eq. 5, the main $j_{\mathrm{LH}}$ peak dislocates markedly more externally (at $r / a \sim 0.9$ ) than in the quasi-linear case of narrow spectrum $\left(r / a \sim 0.5\right.$ for $\left.\Delta n_{/ /} \cong 0.083\right)$, taken as reference. In order to assess the QL effect produced by the RF power density, the $j_{\mathrm{LH}}$ profile has been recalculated assuming a lower launched $p_{\mathrm{RF}}\left(\approx 3 \mathrm{MW} / \mathrm{m}^{2}\right.$ in place of $\left.30 \mathrm{MW} / \mathrm{m}^{2}\right)$. Consequently, the main peak of $j_{\mathrm{LH}}$ dislocates only slightly more externally (from $r / a \sim 0.5$ to $r / a \sim 0.6$ ). Therefore, the effect of a narrow spectrum plays a major role than a larger $p_{\mathrm{RF}}$ in reducing the wave damping, i.e., the former favours more strongly the penetration of the wave packet into hot plasmas, consistently with analytical modelling of Sec. 2.1.

\section{Discussion}

Ray-tracing analysis consists in solving the equation system for the position and wave vector allowing the reconstruction of the wave-phase and the RF power damping rate along the trajectory. The $\mathrm{LH}^{\text {star }}$ tool utilises ray-tracing approach that however respects the geometric limit approximation. Importantly, the latter holds for LH waves propagating far from the cut-off layers located at the plasma edge [10]. Consequently, owing to failure of that limit in the case of weak damping per single pass occurring in most tokamak experiments performed so far, the effect of toroidicity in up-shifting the $n_{/ /}$antenna spectrum is only guessed by this approach, as well originally done for interpreting the outcomes of LHCD experiments. In important experiment of JET of reactorgraded strong absorption per single pass [24,14,15], the geometric limit approximation holds and the $\mathrm{LH}^{\mathrm{star}}$ tool module is able to assess the $j_{\mathrm{LH}}$ profiles without need of any guess. In the $\mathrm{LH}^{\text {star }}$ tool at each radial step, the quasilinear diffusion coefficient is evaluated considering the 
2-D Fokker-Planck relativistic equation solved for the electron distribution function in velocity space, and the quasilinear damping is consistently taken into account in the LH wave power equation. On this basis, the RF power density and LH-wave-driven current density radial profiles are calculated with useful robustness for reactor relevant modelling.

Assuming linear wave theory, Eq. 5, numerical results show that the main $j_{\text {LH }}$ peak dislocates markedly more externally (at $r / a \sim 0.9$ ) than in the QL case of narrow

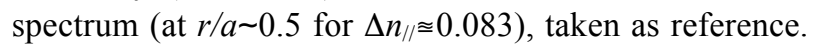
In order to assess the QL effect produced by the RF power density, the $j_{\mathrm{LH}}$ profile has been recalculated assuming a lower launched $p_{\mathrm{RF}}\left(\approx 3 \mathrm{MW} / \mathrm{m}^{2}\right.$ in place of $\left.30 \mathrm{MW} / \mathrm{m}^{2}\right)$. Consequently, the main peak of $j_{\mathrm{LH}}$ dislocates only slightly more externally (from $r / a \sim 0.5$ to $r / a \sim 0.6$ ).

Therefore, the effect of a narrow spectrum plays a major an really exploitable role than a larger $p_{\mathrm{RF}}$ in reducing the wave damping. The former favours more strongly than the latter the penetration of the wave packet into hot plasmas, consistently with analytical modelling of Sec. 2.1, without jeopardising routinary, safe antenna operations. Details on the method and engineering feasibility are discussed in Ref. 9.

\section{Conclusive remarks}

The natural current profiles produced by tokamak plasma are usefully matched by lower hybrid current drive. This was successfully extrapolated to reactor graded high plasma densities [12-15] and, now, also with larger flexibility, to high temperatures envisaged for a thermonuclear reactor $[7,8]$, thus enabling his economic viability.

\section{References}

1. J. Freidberg, J., Plasma Physics and Fusion Energy, (Cambridge Univ. Press, 2007).

2. G. L. Jackson, et al., Regime of very high confinement in the boronized DIII-D tokamak.

3. G. D. Conway, et al., Suppression of Plasma Turbulence During Optimized Shear Configurations in JET, Phys. Rev. Letters 84, 1463 (2000).

4. A. Fasoli, Computational challenges in magnetic-confinement fusion physics, Nature Physics, nphys3744 (2016). G. Giruzzi, et al., Modelling of pulsed and steady-state DEMO scenarios. Nuclear Fusion, 55, Number 7, 073002 (2015).

5. Giruzzi, G., et al., Modelling of pulsed and steady-state DEMO scenarios. Nuclear Fusion, 55, Number 7, 073002 (2015).

6. N. Fisch, Theory of current drive in plasmas, Rev. Modern Physics 59, 175-234 (1987).

7. R. Cesario, et al., Current drive at density required by thermonuclear reactors, Nature Communications, 1 (5) 55 (2010).
8. C. F. F. Karney and N. J. Fisch, Numerical studies of current generation by radio-frequency traveling waves, Phys. Fluids 22, No. 9, 1817 (1979).

9. A. Cardinali et al., to be pub. Scientific Reports

10. A. Cardinali et al., to be pub. on Plasma Phys Control. Fusion

11. M. Brambilla, Kinetic Theory of Plasma Waves (Oxford Press, 1999).

12. S. Bernabei, et al., Lower-Hybrid Current Drive in the PLT Tokamak, Phys. Rev. Letters 49, 1255-1258 (1982).

13. R. Cesario, et al., Spectral broadening of parametric instability in lower hybrid current drive at a high density. Nucl. Fusion 54, 043002 (2014).

14. R. Cesario, et al., Modelling of Lower Hybrid Current Drive in Tokamak Plasmas by including the Spectral Broadening induced by Parametric Instability, Phys. Rev. Letters 9217 175002 (2004).

15. R. Cesario, et al., Spectral broadening of lower hybrid waves produced by parametric instability in current drive experiments on tokamak plasmas, Nucl. Fusion 46, 462-476 (2006).

16. H. Zohm et al., Assessment of the heating and current drive system capabilities for DEMO, Proc. of the $40^{\text {th }}$ European Phys. Society Conf. on Plasma Physics, (2013).

17. C. Gormezano, et al., Lower-hybrid plasma heating via a new launcher - the multijunction grill, Nucl. Fusion 25, 419 (1985).

18. Diamond, P.H., Itoh, S. and Itoh, K., Modern Plasma Physics (Vol.1, Cambridge University Press, 2010).

19. Bonoli, P., and Englade, R.C., "Simulation Model for Lower Hybrid Current Drive", Phys. Fluids 29, 2937 (1986).

20. Landau, L., On the vibration of the electronic plasma, Journal of Physics, Vol X, No. 1, 25 (1946).

21. Diamond, P.H., Itoh, S. and Itoh, K., Modern Plasma Physics (Vol.1, Cambridge University Press, 2010).

22. Drummond W. E. and Pines D., "Non linear stability of plasma oscillations", Nuclear Fusion Suppl., Part 31049 (1962).

23. Peysson, Y., Advances in modeling of lower hybrid current drive, Plasma Physics and Controlled Fusion 58 , 044008 (2016).

24. Crisanti, F., et al., JET Quasistationary InternalTransport-Barrier Operation with Active Control of the Pressure Profile, Phys. Rev. Lett., 88, 145004 (2002). 